CLINICAL STUDY

\title{
Hypoactive sexual desire in transsexual women: prevalence and association with testosterone levels
}

\author{
Els Elaut ${ }^{1}$, Griet De Cuypere ${ }^{1}$, Petra De Sutter ${ }^{3}$, Luk Gijs ${ }^{4}$, Michael Van Trotsenburg ${ }^{4}$, Gunter Heylens ${ }^{1}$, \\ Jean-Marc Kaufman ${ }^{2}$, Robert Rubens ${ }^{1,2}$ and Guy T'Sjoen ${ }^{2}$ \\ ${ }^{1}$ Department of Sexology and Gender Problems, Ghent University Hospital, Belgium, ${ }^{2}$ Department of Endocrinology, Ghent University Hospital Belgium, \\ ${ }^{3}$ Department of Gynaecology, Ghent University Hospital, Belgium and ${ }^{4}$ Center of Gender Dysphoria, VU University Medical Center, Amsterdam, \\ The Netherlands \\ (Correspondence should be addressed to G T’Sjoen; Email: guy.tsjoen@ugent.be)
}

\begin{abstract}
Objective: An unknown proportion of transsexual women (defined as post-operative male-to-female transsexuals on oestrogen replacement) experience hypoactive sexual desire disorder (HSDD). It has been suggested that the absence of ovarian androgen production together with oestrogen treatmentrelated increase in sex hormone-binding globulin (SHBG) levels could be leading to HSDD, due to low levels of biologically available testosterone. This study wishes to document the HSDD prevalence among transsexual women and the possible association to androgen levels.

Design: Cross-sectional study.

Methods: Transsexual women $(n=62)$ and a control group of ovulating women $(n=30)$ participated in this study. Questionnaires measuring sexual desire (sexual desire inventory) and relationship and sexual satisfaction (Maudsley Marital Questionnaire) were completed. Serum levels of total testosterone, LH and SHBG were measured in blood samples obtained at random in transsexual women and in the early follicular phase in ovulating women.

Results: The transsexual group had lower levels of total and calculated free testosterone (both $P<0.001)$ than the ovulating women. HSDD was reported in $34 \%$ of the transsexual and $23 \%$ of the ovulating women $(P=0.30)$. Both groups reported similar levels of sexual desire $(P=0.97)$. For transsexual women, no significant correlation was found between sexual desire and total $(P=0.64)$ or free testosterone $(P=0.82)$. In ovulating women, these correlations were significant $(P=0.006$, resp. $P=0.003)$.

Conclusions: HSDD is reported in one-third of transsexual women. This prevalence is not substantially different from controls, despite markedly lower (free) testosterone levels, which argues against a major role of testosterone in this specific group.
\end{abstract}

European Journal of Endocrinology 158 393-399

\section{Introduction}

Our knowledge on the role of androgens in female sexuality is limited $(1,2)$; many unanswered questions remain concerning the normative data on (free) testosterone across the lifespan. Some studies have observed an association between measures of female sexual desire and serum concentrations of total testosterone and free testosterone $(3,4)$, while others could not confirm this (5). Although a biopsychosocial approach to female sexuality remains in order, there seems to be a consensus upon the role of testosterone in motivational aspects of sexuality such as sexual desire $(6,7)$. Clinicians still wonder under which cut-off levels of free/testosterone complaints of low sexual desire most often occur and a well-defined clinical syndrome is still missing (1). Aware of this debate on the relationship between sexual desire and testosterone, specialists working in centres for treatment of gender dysphoria are confronted with transsexual women (i.e. post-operative male-to-female transsexuals on lifelong oestrogen replacement) complaining of low sexual desire. Assuming sex reassignment surgery is performed skilfully without damage to the neurovascular bundle innervating the neo-clitoris, these complaints might be related to serum androgen levels below the normal female range. Considering a possible role of testosterone in female sexual desire among the general population $(3,4)$, the lack of ovarian androgen production and the effect of continuous oestrogen treatment on the production of sex hormone-binding globulin (SHBG) in transsexual women might lead to hypoactive sexual desire disorder (HSDD) due to low levels of free testosterone. Although an earlier study from our group showed decreased levels of free testosterone in a majority of transsexual women (8), the association between sexual desire and testosterone in this group 
remains to be examined. Considering the observation of sexual desire complaints in transsexual women, and the much debated $(9,10)$ high prevalence of a lack of sexual interest among women in the general population $(25.6 \%$ in Northern European women (11)), it would be relevant to assess this prevalence in transsexual women.

The objective of this study was to assess the prevalence of HSDD and the association between serum androgens and sexual desire in transsexual women. Considering the ongoing debate on both topics in women in general, a control group of community dwelling women was included. The hypothesis was that the transsexual women would have lower serum androgens in comparison with the control group - due to the continuous oestrogen replacement and the lack of an ovarian androgen source. Considering a possible association between androgens and sexual desire, a higher prevalence of HSDD was expected to be seen in the transsexual group.

\section{Materials and methods}

\section{Definitions}

Since the definition of HSDD in the Diagnostic and Stastical Manual of Mental Disorders (DSM-IV) has provoked serious criticism (12), this study set out to work in accordance with the Sexual Function Health Council's consensus definition (13) dated 1998. This interdisciplinary team defined HSDD as the persistent or recurrent deficiency (or absence) of sexual fantasies, thoughts, desire for sexual activity (alone or with a partner) and the inability to respond to sexual cues that would be expected to trigger responsive sexual desire. These symptoms need to be causing personal distress'.

In order to make a correct estimate of the prevalence of HSDD according to the above definition, a distinction was made in the questionnaires between 'spontaneous sexual desire' and 'responsive sexual desire'. This was explained as follows: 'some women experience spontaneous sexual desire (meaning having sexual thoughts, dreams, fantasies or initiating sexual activity). Other women mainly experience sexual desire when approached or touched by their partner or when perceiving sexual stimuli from a book or television, called responsive sexual desire. Another group of women experience both at different times.' Two questions were asked about the frequency of spontaneous and responsive sexual desire (never/rarely, from time to time, often/usually). A third question ('Is your sexual life hampered by problems of sexual desire felt by either yourself or by your partner?') reflected the presence of personal distress caused by potential problems concerning sexual desire. The participants could indicate a problem with too much/little sexual desire in themselves/their partner. We defined the diagnosis of HSDD (nominal variable) as follows: when a participant indicated that she never/rarely experienced either spontaneous or responsive sexual desire, and that her sexual life was hampered by a lack of sexual desire within herself and that this lack caused her distress.

\section{Study population}

Between January and October 2005, 62 transsexual and 30 ovulating women were recruited. Transsexual women on continuous oestrogen replacement were mainly reached through their follow-up visits to the Department of Sexology and Gender Problems (University Hospital Ghent, Belgium) (37\%) and the Center for Gender Dysphoria (VU University Medical Center Amsterdam, The Netherlands) (45.2\%). Since both the Flemish and the Dutch centres work according to similar diagnostic criteria in treating transsexual patients, the comparability of all recruited patients was guaranteed. Eleven transsexual women (17.7\%) responded to banners on transgender community web sites and participated through a local laboratory in The Netherlands. The majority of transsexual women used transdermal oestradiol (mainly in gel form resulting in a $3.0 \mathrm{mg}$ daily dose of oestradiol) or oral oestradiolvalerate (1-4 $\mathrm{mg} ; 40.3$ and $33.9 \%$ ). The remaining participants used oral conjugated oestrogens $(11.3 \%)$ or ethinyloestradiol $(9.7 \%)$.

The control group of ovulating women was recruited through posters in the University Hospital of Ghent and consisted mainly of staff and students. Eligible participants were healthy women between 18 and 45 years of age. Exclusion criteria were use of steroidal contraception (combination pill, mini-pill, vaginal ring, contraceptive patch or progestin intrauteine device), medication possibly influencing androgen levels or sexual desire (like antidepressants or glucocorticoids) and other confounders for SHBG, such as alcoholism, cirrhosis, Cushing syndrome, and hyper- and hypothyroidism (14). We chose not to include isogender women who have been oophorectomized and treated with oestrogens in our control group as this is the first study to address sexual desire in relation to androgens in transsexuals and we aimed to describe the difference in androgen status between groups caused by the presence/absence of ovaries. Table 1 shows characteristics of both groups. Because of the age criterion in the ovulating women, both groups differed significantly in age $(P<0.001)$.

\section{Study procedures}

A web site was designed where participants read about the background and objectives of the study to guarantee an informed consent. They completed questionnaires on sexual desire and relationship/sexual satisfaction online and subsequently contacted the research team for the withdrawal of a morning blood sample. A minority of the Dutch transsexual women received a package which would allow their general practitioner or a local clinical laboratory to draw a morning blood sample. 
Table 1 Characteristics of participants.

\begin{tabular}{|c|c|c|c|}
\hline Characteristic & Transsexual women $(n=62)$ & Ovulating women $(n=30)$ & Significance $^{a}$ \\
\hline \multicolumn{4}{|l|}{ Age (year) } \\
\hline $\begin{array}{l}\text { Mean } \pm \text { S.D. } \\
\text { Range }\end{array}$ & $\begin{array}{c}43.0 \pm 10.0 \\
22-65\end{array}$ & $\begin{array}{c}31.0 \pm 7.1 \\
19-45\end{array}$ & $<0.001$ \\
\hline Body mass index $\left(\mathrm{kg} / \mathrm{m}^{2}\right)$ & & & 0.12 \\
\hline Mean \pm S.D. & $24.1 \pm 4.7$ & $22.8 \pm 4.3$ & \\
\hline Range & $15.2-38.6$ & $18.4-38.4$ & \\
\hline \multicolumn{4}{|l|}{ Sex reassignment surgery (year) } \\
\hline Time since surgery & $5.2 \pm 5.8$ & & \\
\hline Duration of oestrogen replacement & $7.4 \pm 5.8$ & & \\
\hline
\end{tabular}

${ }^{a}$ Significance levels are according to non-parametric Mann-Whitney $U$ tests.

This package contained all necessary medical material as well as the instructions for the participant and their general practitioner or local laboratory to perform the blood draw correctly and without any cost. After the blood collection, serum and red blood cells were separated locally. A courier company immediately transported the serum to the University Hospital of Ghent (Belgium) where it was stored at $-80{ }^{\circ} \mathrm{C}$ until analysis. Most transsexual women, however, were recruited at their follow-up visits to one of the two participating centres and had a venous blood sample collected between 0800 and $1200 \mathrm{~h}$ at their centre. They subsequently filled out the questionnaires online. All transsexual women had their blood sample drawn at random, regardless of the timing of oestrogen intake. All Flemish ovulating women had their blood drawn between 0800 and $1200 \mathrm{~h}$ in the early follicular phase (third or fourth day after the start of menstruation).

This study complied with the recommendations of the Declaration of Helsinki and was approved by the Ethical Committee of the Ghent University Hospital.

\section{Measures}

Sexual desire The Dutch version of the 14-item sexual desire inventory (15) was used to measure sexual desire, defined as 'an interest in sexual activity', as a cognitive variable. The scale measures the frequency and strength of thoughts in regards to seeking out or being receptive to sexual stimuli. For the frequency-items, participants chose one out of the seven options. For the strengthitems, participants scored their sexual desire on a 9-point Likert scale ranging from 0 (no desire) to 8 (strong desire). The participants were asked to take the previous month as a reference. Adding items resulted in a score for dyadic sexual desire (interest in behaving sexually with a partner) and solitary sexual desire (interest in behaving sexually by oneself). The sexual desire inventory has a good reliability and validity (15). Internal consistency was high, in the group of transsexual (Cronbach's $\alpha=0.93$ ) and ovulating women (Cronbach's $\alpha=0.92$ ).
Sexual and relationship satisfaction The Dutch version of the 20-item Maudsley Marital Questionnaire (16) was used to measure relationship, sexual and general life satisfaction. Only participants with a steady partner filled it out. Every item is scored on a Likert scale from 0 to 8 . The participants were asked to take the previous 2 weeks as a reference. On all subscales, a higher score indicates more problems. The Maudsley Marital Questionnaire has good reliability and validity (15). The internal consistency was high as well in the group of transsexual (Cronbach's $\alpha=0.84$ ) and ovulating women (Cronbach's $\alpha=0.87$ ).

Diagnosis of HSDD At the time this study was conducted, no validated questionnaires were available to establish an HSDD diagnosis. Working with the consensus definition by Basson et al. (13), a very strict adaptation was made as described above.

Hormone assays Serum testosterone was assayed in 2 ml serum samples with an in-house RIA in duplicate after ether extraction followed by paper chromatography $(17,18)$; the intra- and inter-assay coefficients of variation for this assay were $5.6 \%$ (at a concentration of $35 \mathrm{ng} / \mathrm{dl}$, i.e. $1.21 \mathrm{nmol} / \mathrm{l}$ ) and $8.5 \%$ (at a concentration of $25 \mathrm{ng} /$ dl, i.e. $0.87 \mathrm{nmol} / \mathrm{l}$ ) respectively and the lower limit of quantification was $2 \mathrm{ng} / \mathrm{dl}(0.07$ $\mathrm{nmol} / \mathrm{l})$. Commercial kits for RIA were used to determine the serum concentrations of SHBG (Orion Diagnostica, Espoo, Finland) and DHEA sulphate (DSL Inc., Webster, TX, USA); the intra-assay coefficients of variation were between 2.5 and $8.3 \%$ and between 3.1 and $5.6 \%$ for SHBG and DHEA-S respectively. For all measurements, all samples from transsexual women and controls were assayed in a same assay run. Serum free testosterone was calculated from the total serum hormone concentration, serum SHBG and serum albumin, using a validated equation derived from the mass action law (19). 


\section{Statistical analysis}

Since the distribution of the dependent variables (sexual desire, sexual/relationship satisfaction) did not follow a normal distribution, non-parametric Mann-Whitney $U$ tests were used to test between-group differences of these ordinal variables. A $\chi^{2}$ test was used to compare the nominal variable (diagnosis of HSDD) between both groups. Partial correlations tested the association between levels of testosterone and sexual desire.

\section{Results}

\section{Sexual desire}

All participants completed the sexual desire inventory reflecting solitary and dyadic sexual desire. As shown in Table 2, there were no significant differences between ovulating and transsexual women, either in solitary sexual desire $(P=0.97)$ or in dyadic sexual desire $(P=0.26)$.

\section{Relationship/sexual satisfaction}

One-sample $t$-tests showed that the control group did not differ significantly from the normal female population (18) (relationship satisfaction: $t(20)=-0.18$, $P=0.86$; sexual satisfaction: $t(20)=0.50, P=0.62$; general life satisfaction: $t(20)=0.11, P=0.91)$, confirming its representativeness. Transsexual women reported significantly less sexual satisfaction $(P=0.002)$ than ovulating women. Regarding relationship and general life satisfaction $(P=0.82$ and $P=0.55)$, transsexual and ovulating women did not differ significantly.

\section{Prevalence of HSDD}

HSDD was diagnosed in $33.9 \%$ of transsexual and in $23.3 \%$ of ovulating women. Although the percentage is

Table 2 Psychosexual outcomes.

\begin{tabular}{lccc}
\hline Variable & $\begin{array}{c}\text { Transsexual } \\
\text { women }\end{array}$ & $\begin{array}{c}\text { Ovulating } \\
\text { women }\end{array}$ & Significance $^{\mathrm{a}}$ \\
\hline $\begin{array}{c}\text { Sexual desire } \\
\text { inventory }\end{array}$ & $(n=62)$ & $(n=30)$ & \\
$\begin{array}{c}\text { Solitary sexual } \\
\text { desire }\end{array}$ & $9.2 \pm 6.2$ & $9.2 \pm 6.2$ & 0.97 \\
$\begin{array}{c}\text { Dyadic sexual } \\
\text { desire }\end{array}$ & $29.2 \pm 14.8$ & $32.7 \pm 14.0$ & 0.26 \\
$\begin{array}{c}\text { Maudsley Marital } \\
\text { Questionnaire }\end{array}$ & $(n=27)$ & $(n=21)$ & \\
$\begin{array}{c}\text { Relationship } \\
\text { satisfaction }\end{array}$ & $11.3 \pm 11.8$ & $10.5 \pm 8.3$ & 0.82 \\
$\begin{array}{c}\text { Sexual } \\
\text { satisfaction }\end{array}$ & $18.0 \pm 9.4$ & $6.2 \pm 8.8$ & 0.002 \\
$\begin{array}{c}\text { General life } \\
\text { satisfaction }\end{array}$ & $8.7 \pm 6.3$ & $7.3 \pm 2.7$ & 0.55 \\
\hline
\end{tabular}

Data are presented as mean \pm s.D.

${ }^{a}$ Significance levels are according to non-parametric Mann-Whitney $U$ tests. higher in the transsexual group, there was no difference in the prevalence of $\operatorname{HSDD}\left(\chi^{2}(1)=1.06, P=0.30\right)$ between both the groups. Our hypothesis concerning the frequency of HSDD was thus not confirmed. Furthermore, these percentages did not differ significantly from the general female population $(25.6 \%)$ (11) (95\% confidence interval, 22.1-45.7\%).

\section{Hormonal data}

In Table 3, serum concentrations of SHBG, DHEA-S, free testosterone and total testosterone are presented. In both the groups, all concentrations were as expected physiologically. Our hypothesis concerning the serum concentrations of androgens was thus confirmed. The levels of total testosterone and calculated free testosterone were significantly (both $P<0.001$ ) lower in transsexual women when compared with those of the ovulating women. More specifically, $66.1 \%$ of transsexual women had levels of total testosterone below P25 in ovulating women $(\mathrm{P} 25=23.88 \mathrm{ng} / \mathrm{dl}$; $0.8 \mathrm{nmol} / \mathrm{l})$. For calculated free testosterone, $59.7 \%$ of transsexual women had levels below P2 5 in ovulating women (P25=0.28 ng/dl; 0.01 $\mathrm{nmol} / \mathrm{l})$. Two ANCOVAs showed that these lower levels were a main effect of group and not of the higher age of the transsexual group $(F(1)=12.573, P=0.001$ for total testosterone and $F(1)=9.235, P=0.003$ for free testosterone). Serum levels of SHBG $(P=1.00)$ and DHEA-S $(P=0.57)$ did not differ between both the groups.

Transsexual women using oral preparations of oestrogen replacement had significantly higher levels of SHBG than those using transdermal preparations $(73.3 \pm 45.1 \mathrm{nmol} / \mathrm{l}$ versus $47.5 \pm 23.7 \mathrm{nmol} / \mathrm{l}$; $P=0.02$ ). Levels of total testosterone and calculated free testosterone did not differ between groups according to the mode of administration (data not shown). Differences in serum concentrations (and psychosexual measures) between different types of oral substitution (ethinyloestradiol, conjugated oestrogens and oestradiolvalerate) could not be tested due to low numbers in some groups.

\section{Association between sexual desire and hormone levels}

With the age of the transsexual women being higher than those of the ovulating women, and the known agerelated decline in levels of DHEA-S $(20,21)$, the correlations between androgen levels and measures of sexual desire (Table 4) have been corrected for age by using partial correlations. In transsexual women, no significant relation was found between androgen levels and measures of sexual desire. However, in the ovulating women a significant positive correlation was found between solitary sexual desire and levels of total testosterone $(r=0.50, P=0.006)$ and free testosterone $(r=0.53, P=0.003)$. In both the groups, no significant differences in serum concentrations of DHEA-S, 
Table 3 Serum concentrations in transsexual and ovulating women.

\begin{tabular}{|c|c|c|c|c|}
\hline & $\begin{array}{c}\text { Transsexual } \\
\text { women }(n=62)\end{array}$ & $\begin{array}{c}\text { Ovulating } \\
\text { women }^{\mathrm{a}}(n=30)\end{array}$ & $\begin{array}{c}\text { Normal } \\
\text { female range }\end{array}$ & Significance $^{c}$ \\
\hline \multicolumn{5}{|l|}{ TT (ng/dl) } \\
\hline Mean \pm S.D. & $20.0 \pm 9.6$ & $33.9 \pm 17.9$ & & $<0.001$ \\
\hline Range & $2.4-53.0$ & $16.6-109.1$ & $10.0-80.0$ & \\
\hline \multicolumn{5}{|l|}{ FT (ng/dl) } \\
\hline Mean士s.D. & $0.26 \pm 0.16$ & $0.47 \pm 0.31$ & & $<0.001$ \\
\hline Range & $0.03-0.99$ & $0.19-1.61$ & $0.20-0.50$ & \\
\hline \multicolumn{5}{|l|}{ SHBG (nmol/l) } \\
\hline Mean \pm s.D. & $63.1 \pm 38.1$ & $55.7 \pm 19.9$ & & 1.00 \\
\hline Range & $20.7-197.6$ & $17.7-98.1$ & $15.5-114.0$ & \\
\hline \multicolumn{5}{|l|}{ DHEA-S $(\mu \mathrm{g} / \mathrm{dl})$} \\
\hline Mean \pm S.D. & $219.5 \pm 115.9$ & $228.8 \pm 88.5$ & & 0.57 \\
\hline Range & $5.5-536.5$ & $94.9-409.0$ & $98.8-340.0$ & \\
\hline
\end{tabular}

aEarly follicular values.

${ }^{\mathrm{b}}$ Reference ranges according to local laboratory.

${ }^{\mathrm{c}}$ Significance values are for comparison between the transsexual and ovulating women according to non-parametric Mann-Whitney $U$ tests.

Total testosterone, TT; Free testosterone, FT; Sex hormone-binding globulin, SHBG.

total testosterone or free testosterone were found between participants with and without HSDD (data not shown). In the transsexual women, there was no relation between having an HSDD diagnosis and having a free testosterone level below P25 in ovulating women $\left(\chi^{2}(1)=0.25, P=0.61\right)$.

\section{Discussion}

This study is to our knowledge the first to address sexual desire and serum androgens in a relatively large group of transsexual women. Using a strict measure of HSDD, one out of three transsexual women suffers from a lack of sexual desire after sex-reassignment surgery. Since research concerning HSDD prevalence in this group has not yet been reported, comparisons with other samples are not possible. HSDD does not appear to be substantially more prevalent in this group than in the general female population.

Transsexual women experience sexual desire of similar frequency and intensity as ovulating women. Nevertheless, there seems to be a general 'sexual discontent' in transsexual women such as the one that can be gathered from the lower sexual satisfaction this group experiences in its sexual partnerships. Since sexual satisfaction was not the focus of this study, we were not able to explore the reasons behind this.

The serum concentrations of DHEA-S are comparable in transsexual and ovulating women, indicating a similar level of adrenal androgen production. Serum concentrations of total testosterone measured by RIA following extraction and chromatographic separation and calculated free testosterone are, as expected, markedly lower in the transsexual women. In $59.7 \%$ of the transsexual women, concentrations remain below P25 of free testosterone found in ovulating women. In an earlier study, $32.1 \%$ of transsexual women had serum levels of free testosterone within the expected female range (direct RIA method used) (8). According to the same criterion, this is $35.5 \%$ in the present study.

In the transsexual women, we could not establish an association between sexual desire and androgens. In the ovulating women, a positive association was observed: higher serum concentrations of total testosterone and free testosterone accompany a higher frequency and intensity of solitary sexual desire. This result is in line with observational research in premenopausal women where an association exists between free testosterone and sexual desire (3). The absence of this association in

Table 4 Partial correlations between sexual desire measures and hormone concentrations.

\begin{tabular}{lccrrr}
\hline & \multicolumn{2}{c}{ Transsexual women $(n=62)$} & & \multicolumn{2}{c}{ Ovulating women $(n=30)$} \\
\cline { 2 - 3 } \cline { 5 - 6 } Hormone & Solitary sexual desire & Dyadic sexual desire & & Solitary sexual desire & Dyadic sexual desire \\
\hline & $r(P)$ & $r(P)$ & $r(P)$ & $r(P)$ \\
DHEA-S & $0.07(0.59)$ & $0.13(0.34)$ & & $0.20(0.29)$ & $0.35(0.06)$ \\
TT & $-0.06(0.64)$ & $-0.03(0.83)$ & & $0.50(0.006)^{\mathrm{a}}$ & $0.29(0.13)$ \\
FT & $0.03(0.82)$ & $0.10(0.42)$ & & $0.53(0.003)^{\mathrm{a}}$ & $0.33(0.08)$ \\
\hline
\end{tabular}

All correlations are corrected for age.

${ }^{a}$ Values lower than the $5 \%$ level of significance.

Total testosterone, TT; Free testosterone, FT. 
transsexual women could have several reasons. First, the majority has very low levels of (free) testosterone. This complicates finding a relation with sexual desire. Second, the profound decrease in testosterone levels transsexual women experienced during hormonal and surgical sex reassignment could play a role. It is possible that such a decrease demands a certain 'sexual reconditioning' from the individual and the partnership. Research on surgical menopausal women has described - if to a lesser extent - somewhat similar events; removal of the ovaries causes a decrease in testosterone levels and sexual desire (22-25). This psychological process of adjusting to a lower serum level could be of influence on the association between testosterone and sexual desire. Third, not only androgens play a role in sexual motivation. It is very well possible that the relief of finally being in the desired body and being socially accepted as a woman causes a healthy sexual appetite despite very low testosterone levels. Fourth, the functionality and aesthetics of the new genitals could have their influence on sexual satisfaction and possibly on sexual desire.

As to the limitations of this study, we have no information on the used method of non-hormonal contraception in the ovulating group, making it impossible to investigate this potential influence on sexual desire. Also, the subtle nuances of transsexual women's sexual desire might not have been adequately measured as the questionnaires were designed for women in general, even though they did allow detection of differences in sexual satisfaction. Furthermore, despite the fact that we were able to study a substantial number of transsexual women, and that the findings for our control group are in good agreement with those for the general population, the power of the comparison between transsexual and control women was limited. Nevertheless, the observations in this study converge to indicate that low testosterone does not play a prominent role in low sexual desire in transsexual women; i.e. a markedly high prevalence of low (free) testosterone in the transsexual women is accompanied by an at most modest increase of the prevalence of HSDD, whereas within this group, there is no relationship between androgen levels and indices of sexual desire. Measurement of low testosterone in women is technically difficult and has been an impediment in defining a clinical syndrome of androgen deficiency in women (1). In the present study, we addressed this technical problem by extracting testosterone from an adequately large, $2 \mathrm{ml}$ serum sample and using a well-validated method involving a chromatographic separation step.

In conclusion, HSDD is reported in one-third of transsexual women. This prevalence is not substantially different from controls and reports from the general population. The levels of (free) testosterone in transsexual women were found to be at or below the lower end of the spectrum for (free) testosterone seen in the control group. No apparent association exists between these levels and the complaints of low sexual desire patients experience, which argues against a major role of (free) testosterone in this specific group. These observations should therefore be broadened by studies in the psychological and social field to help explain possible mediating factors.

\section{Acknowledgements}

The authors are indebted to Jos Megens and Kaatje Toye for their help and expert assistance in the completion of this study. P D S is holder of a fundamental clinical research mandate by the Flemish Foundation for Scientific Research (FWO-Vlaanderen).

\section{References}

1 Wierman ME, Basson R, Davis SR, Khosla S, Miller KK, Rosner W \& Santoro N. Androgen therapy in women: an endocrine society clinical practice guideline. Journal of Clinical Endocrinology and Metabolism 200691 3697-3710.

2 Scepkowski LA, Georgescu M \& Pfaus JG. Neuroendocrine factors in sexual desire and motivation. In Women's Sexual Function and Dysfunction. Study, Diagnosis and Treatment, pp 159-167. Eds I Goldstein, CM Meston, SR Davis \& AM Traish, London: Taylor \& Francis, 2006.

3 Turna B, Apaydin E, Semerci B, Altay B, Cikili N \& Nazli O. Women with low libido: correlation of decreased androgen levels with female sexual function index. International Journal of Impotence Research 200517 148-153.

4 Santoro N, Torrens J, Crawford S, Allsworth JE, Finkelstein JS, Gold EB, Korenman S, Lasley WL, Luborsky JL, McConnell D, Sowers MF \& Weiss G. Correlates of circulating androgens in mid-life women: the Study of Women's Health Across the Nation. Journal of Clinical Endocrinology and Metabolism 200590 4836-4845.

5 Davis SR, Davison SL, Donath S \& Bell RJ. Circulating androgen levels and self-reported sexual function in women. Journal of the American Medical Association 2005294 91-96.

6 Sherwin BB, Gelfand MM \& Brender W. Androgen enhances sexual motivation in females: a prospective, crossover study of sex steroid administration in the surgical menopause. Psychosomatic Medicine 198547 339-351.

7 Sherwin B. Changes in sexual behavior as a function of plasma sex steroid levels in post-menopausal women. Maturitas 19857 225-233.

8 De Cuypere G, T'Sjoen G, Beerten R, Selvaggi G, De Sutter P, Hoebeke P, Monstrey S, Vansteenwegen A \& Rubens R. Sexual and physical health after sex reassignment surgery. Archives of Sexual Behavior 200534 679-690.

9 Bancroft J, Loftus J \& Long JS. Distress about sex: a national survey of women in heterosexual relationships. Archives of Sexual Behavior 200332 193-208.

10 Rosen RC \& Laumann EO. The prevalence of sexual problems in women: how valid are comparisons across studies? Commentary on Bancroft, Loftus, and Long's 'distress about sex: a national survey of women in heterosexual relationships' Archives of Sexual Behavior 200332 209-211.

11 Laumann EO, Nicolosi A, Glasser DB, Paik A, Gingell C, Moreira E \& Wang T. Sexual problems among women and men aged 40-80 y: prevalence and correlates identified in the Global Study of Sexual Attitudes and Behaviors. International Journal of Impotence Research $20051739-57$. 
12 Basson R. Are our definitions of women's desire, arousal and sexual pain disorders too broad and our definitions of orgasmic disorder too narrow? Journal of Sex and Marital Therapy 2002 28 289-300.

13 Basson R, Berman J, Burnett A, Derogatis L, Ferguson D, Fourcroy J, Goldstein I, Graziottin A, Heiman J, Laan E, Leiblum S, Padma-Nathan H, Rosen R, Segraves K, Segraves RT, Shabsigh R, Sipski M, Wagner G \& Whipple B. Report of the international consensus development conference on female sexual dysfunction: definitions and classifications. Journal of Urology $2000 \mathbf{1 6 3}$ 888-893.

14 Wu F, Ames R, Evans MC, France JT \& Reid IR. Determinants of sex hormone-binding globulin in normal postmenopausal women. Clinical Endocrinology 200154 81-87.

15 Spector IP, Carey MP \& Steinberg L. The sexual desire inventory: development, factor structure, and evidence of reliability. Journal of Sex and Marital Therapy 199622 175-190.

16 Arrindell WA, Boelens W \& Lambert H. On the psychometric properties of the Maudsley Martial Questionnaire (MMQ): evaluation of self-ratings in distressed and 'normal' volunteer couples based on the Dutch version. Personality and Individual Differences 19834 293-306.

17 Vermeulen A \& Verdonck L. Radioimmunoassay of $17 \beta$-hydroxy$5 \alpha$-androstan-3-one, 4-androstene-3, 17-dione, dehydroepiandrosterone, 17-hydroxyprogesterone and progesterone and its application to human male plasma. Journal of Steroid Biochemistry 19767 $1-10$.

18 Vermeulen A \& Verdonck L. Sex hormone concentrations in postmenopausal women. Relation to obesity, fat mass, age and years menopause. Clinical Endocrinology 19789 407-412.

19 Vermeulen A, Verdonck L \& Kaufman JM. A critical evaluation of simple methods for the estimation of free testosterone in serum. Journal of Clinical Endocrinology and Metabolism $1999 \mathbf{8 4}$ 3666-3672.
20 Laughlin GA, Barret-Conner E, Kritz-Silverstein D \& von Muhlen D. Hysterectomy, oophorectomy, and endogenous sex hormone levels in older women: the Rancho Bernardo study. Journal of Clinical Endocrinology and Metabolism 200085 645-651.

21 Randolph JF Jr, Sowers M, Gold EB, Mohr BA, Luborsky J, Santoro N, McConnell DS, Finkelstein JS, Korenman SG, Matthews KA, Sternfeld B \& Lasley BL. Reproductive hormones in the early menopausal transition: relationship to ethnicity, body size, and menopausal status. Journal of Clinical Endocrinology and Metabolism $2003 \mathbf{8 8} 1516-1522$.

22 Nathorst-Böös J, von Schoultz B \& Carlström K. Elective ovarian removal and oestrogen replacement therapy - effects on sexual life, psychological well-being and androgen status. Journal of Psychosomatic Obstetrics and Gynaecology $199314283-293$.

23 Mazer NA, Leiblum SR \& Rosen RC. The brief index of sexual functioning for women (BISF-W): a new scoring algorithm and comparison of normative and surgically menopausal populations. Menopause 20007 350-363.

24 McHorney CA, Rust J, Golombok S, Davis S, Bouchard C, Brown C, Basson R, Sarti CD, Kuznicki J, Rodenberg C \& Derogatis L. Profile of female sexual function: a patient-based, international, psychometric instrument for the assessment of hypoactive sexual desire in oophorectomised women. Menopause 200411 474-483.

25 Dennerstein L, Koochaki P, Barton I \& Graziottin A. Hypoactive sexual desire disorder in menopausal women: a survey of Western European women. Journal of Sexual Medicine 20063 212-222.

Received 8 November 2007

Accepted 25 November 2007 